\title{
Environmental monitoring of urban areas by using a remote sensor network
}

\author{
V. Ferrara ${ }^{1}$, G. Filice ${ }^{2}$ \& C. M. Ottavi ${ }^{1}$ \\ ${ }^{I}$ Department of Electronic Engineering, Sapienza University of Rome, \\ Italy \\ ${ }^{2}$ Comtest wireless S.r.l., Turin, Italy
}

\begin{abstract}
Planning of sustainable development in a city depends on a lot of factors: policy, economy advantages, social justice and environmental safeguard. Technology evolution in the field of science can help in achieving one's aim. This paper concentrates the analysis on some new technologies of ICT (Information and Communications Technologies) activity. These, together with electronic nanotechnology development, allow more efficient management of data and tools that are indispensable for an efficient plan. WiFi and WiMAX (standards of wireless communications) devices, the Internet and GIS as Terrapack ${ }^{\circledR}$ are fundamental for developing a modern architecture of environmental management. Sensors distributed on the territory of an urban area define the WSN (Wireless Sensor Network). They use Internet Protocol (IP) for the link to the co-ordination station (central or local station): the SNS (Sensor Network Server). IP addressing allows device setup and the use of an Internet connection. This one increases enormously the number of potential data which can be included in the algorithms of environmental evaluations. We designed and tested the prototype architecture by use of LAN (Local Area Network) for controlling some detection sensors and standard monitoring devices such as a webcam. The WLAN (Wireless LAN) version of this architecture is carried out immediately in the case of a small monitoring area. A metropolitan area needs new technologies that are in the experimental phase or submitted to policy convenience.
\end{abstract}

Keywords: monitoring, sensors, GIS, WSN, WiMax, WiFi. 


\section{Introduction}

Many urban areas continuously monitor physical quantities or other parameters and check for environmental hazards. These measures represent fundamental data that are used in the prevention activity of residents' health risks. A typical example is the daily control of air pollution. Monitoring is carried out by means of detection stations, fixed or mobile [6], as nodes of a network distributed in the territory. Data are collected in historical software files, which allow in the next time the achievement of urban maps, where the overcoming of risk threshold is put in evidence.

Moreover in general, many different services of public utility need the acquisition of data that are spread in urban area.

Emergency management demands acquisition, data elaboration and visualization in real time mode. Typically, fire-brigade department coordinates a lot of remote operative centres and it manages many dynamical data during disaster event.

As other example, transport sector can obtain advantages from detection of indicators that define: traffic state, temporary one-way only, special events that stop cars by involving some streets, etc.

This paper aims to demonstrate how much the new technologies are modifying the design of a monitoring structure and how much they add sources of potential data increasing reliability of elaborated results.

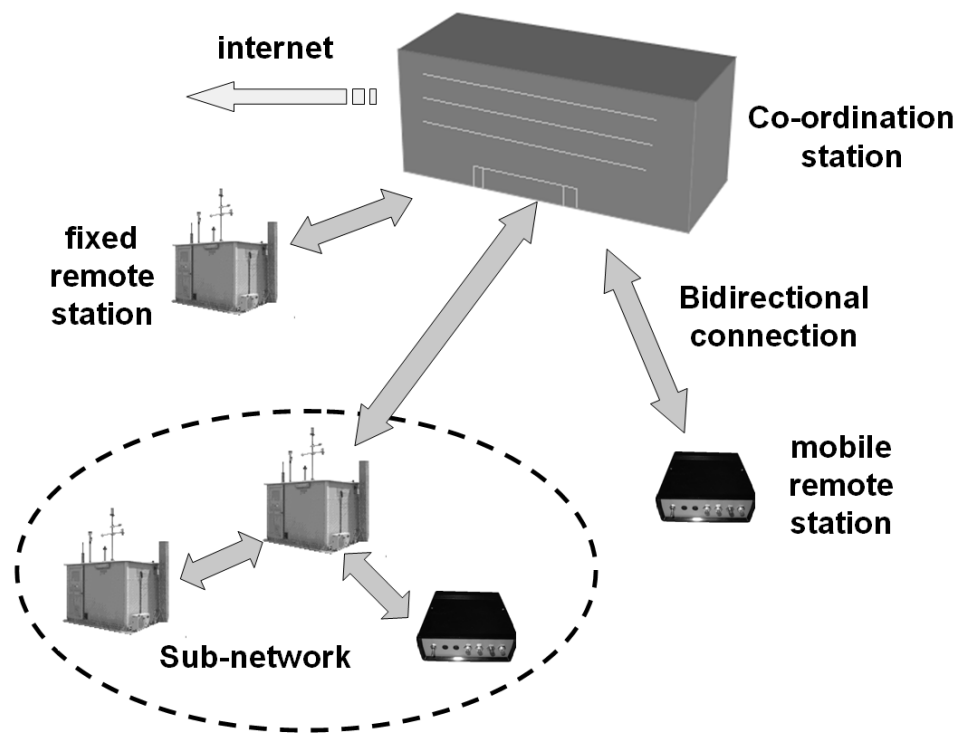

Figure 1: An efficient monitoring structure uses different sensors inside fixed and mobile stations distributed in the territory. 
Some technology will be really usable only in the next years, because commercial policy put off its introduction or because it cannot carry out an important role into the wider trade at moment. This is for example the case of devices developed by using of WiMAX standard [12].

The view is to evolve towards futuristic technologies starting from compatible standard (as it is declared). The sustainability of these developments and researches is joined with the subject of the projects that concerns with sustainable development of urban area.

An example of our research target is the monitoring of air pollution inside a city. Software simulations, data collection, data fusion and prediction results are made by central station of co-ordination and eventually pre-elaborated by some remote detection stations. These ones are ground-stations, normally at fixed location. They measure meteorological and pollutants concentration realizing onsite monitoring network [13]. Moreover they can be equipped with webcam and other sensors controlling the motor traffic. We consider small devices that include some sensors also as stations. Their main characteristics are portability and a mobile service of measure. All stations are assimilated as nodes of a global network. Fixed or mobile state determines the selection of sub-network connection: wire, wireless, point-to-point link, broadcast. No negligible advantages can be obtained by using of Internet. Limitations of the number of nodes could be imposed by communication technology. At the same mode, a specific technology has restriction of the service area size. Figure 1 shows a typical organization of sensors and stations.

In the next section we will examine different technologies for local and metropolitan area service.

\section{Urban network of remote sensors}

In all environmental monitoring cases (air pollution, emergency management, transport sector, etc.) a lot of data of different origin and kind are detected, collected and transmitted to a coordination main station. This one organizes nonhomogenous data producing new global parameters, the indicators, and it elaborates a strategy of intervention.

In some case remote detection station pre-elaborates raw data. Elementary processing is to joint different data that have common characteristics of: space, time and other quantity.

\subsection{Network and mobile nodes}

Location is whatever the fundamental element of data aggregation in view of the map implementation. For fixed station, one measure of location is carried out at its installation. In the case of mobile, each station includes a satellite navigation system as GPS and Galileo in the future. Precision of the location measure can demand integrate system (as inertial integration) or more precise differential measurement [8]. The last one computes location by means of relative position of mobile from one reference station. Usually the reference station transmits 
correction data to apply in the computation algorithm using the broadcast. RTCM-104 (Radio Technical Commission for Maritime Services) [7] is the serial protocol used for broadcasting pseudo range corrections from DGPS (Differential-GPS) reference stations. Recent development uses the Internet Protocol as carrier of real time corrections: NTRIP (Networked Transport of RTCM via Internet Protocol) [9]. It has been developed by the Federal Agency for Cartography and Geodesy of Germany and it enables the streaming of DGPS or RTK (Real-Time Kinematic) correction data via the Internet using GPRS or other technologies.

Single sensor can transmit directly its measure to main station A cluster of different sensors, managed by a fixed or mobile detection station, are preelaborated before the transmission. More techniques can be used to link different sources and central station (the co-ordination station), saving identification code brought from each source.

This one (or remote station) is a specific node of a spatial network, generally no fixed. Single node is unique with peculiar feature and its characteristic is listed in the dynamic archive of central station. Consequently a structure oriented to solve the problems of this network should be LAN ((Local Area Network) or MAN (Metropolitan Area Network). LANs use certain specific connectivity technologies, primarily Ethernet and Token Ring. MAN connects an area larger than a LAN but smaller than a WAN (Wide Area Network), such as a city. Moreover the physical connectivity should be wireless (WLAN).

\subsection{Wireless}

A lot of operative structures use WiFi (Wireless-Fidelity): the standard IEEE 802.11x [10]. This is the case of a few nodes and of short distance between central station and transmitters of the remote sensors. In effect, WiFi continues to be the pre-eminent technology for building general-purpose wireless networks. Maximum radius of service coverage is a few kilometres by using of isotropic and directive antennas, named access points. Usually, in the case of medium area, such as a district of the city, the obstacles weaken the signal and an increase of the access points are called for. Consequently, when one considers a network extended on a wide urban area, MAN should be taken: HiperMAN (High Performance Radio Metropolitan Area Network), HiperLAN, WiMax (Worldwide Interoperability for Microwave Access).

HiperLAN has been defined by ETSI (European Telecommunications Standards Institute) and it represents the European alternative in competition to USA's WLAN (IEEE 802.11). It uses: standards ETS 300652 and ETS 300 893 , low power of emission ( $\approx 1$ Watt), $40 \mathrm{~km}$ of maximum distance, $54 \mathrm{Mbit} / \mathrm{sec}$ at $5 \mathrm{GHz}$ of frequency. HiperMAN is the European standard carried out by ETSI and it is the alternative to WiMAX [11] in the range of frequency 2-11 GHz.

WiMax devices are in conformity with IEEE 802.16 standard. This technology can connect to Internet the WiFi hotspot. Coverage is 9,300 square $\mathrm{km}$ (about $50 \mathrm{~km}$ of radius) for also NLOS (Non Line of Sight) link.

WiMAX is convenient from the point of view of mobile service and its technology is in progress; actually size of employed transmitter is big, power 
supply is high and no suitable for portable devices. Nevertheless, the decrease of integration scale predicts advantage of this technology in next near years.

Mobile connectivity of WiMAX is allowed by the radio frequencies: $2.3,2.5$, 3.3, 3.5 and $5.8 \mathrm{GHz}$. WiMAX Forum recommended better radio frequency allocation at 5.5-5.69 GHz.

For Italian country, WiMAX is in the experimental phase using the band 3.4$3.6 \mathrm{GHz}$.

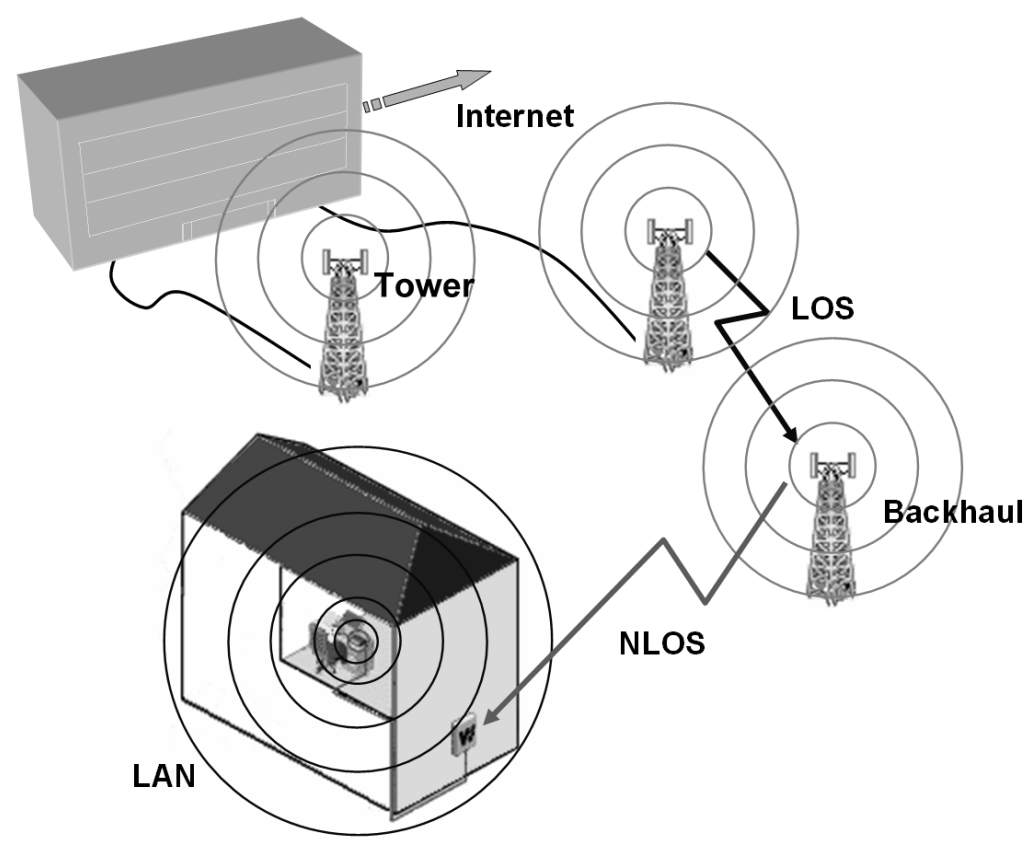

Figure 2: $\quad$ Structure of WiMAX connections.

A WiMAX system consists of two parts: tower and WiMAX receiver. Moreover, it can provide two forms of wireless service: NLOS (lower frequency range 2-11 GHz: licensed bands)) and LOS (higher frequency range 10-66 GHz: normally unlicensed bands).

One WiMAX tower can cover a very large area, it can connect directly to the Internet using a high-bandwidth and it can connect to another WiMAX tower (often referred to as a backhaul) using a LOS (Line-of-Sight) link.

Receiver can be: a small box including antenna, a PCMCIA card or it can be built into a laptop.

Figure 1 shows a typical scenario of WiMAX connectivity.

\subsection{Standard opportunity}

Obviously a traditional analog radio link can be used to solve problem of data transmission between remote and main station. But WLAN allows more 
advantages in order to: identification and addressing of network nodes, bidirectional communication between two nodes and direct connectivity to Internet. The last one is very important feature allowing: the implementation of sophisticated pre-elaborations that use data to be found by WEB; precise position measurement by means of NTRIP method, etc.

Network architecture can include WiFi subsystems. Each of these is identified as a global node (hotspot) included inside the bigger WiMAX network. So, a remote station: coordinates and controls different addressable sensors distributed in a local area, it pre-elaborates data, detected from sensors, together with other data received by WEB and it realizes the link to central station by WiMAX.

From the point of view of interface, operator uses a transparent IP (Internet Protocol) addressing without specifying the layer WiMAX or WiFi.

In the case of traditional analog radio link, designer works outside the conventional standards and he must adapt the specific network structure. Whereas this is a good quality of WiFi-WiMAX standard opening on to a wide world of shared data.

WiMAX isn't licensed freely in Italian country: frequency range $3.4-3.6 \mathrm{GHz}$ has been assigned to a few electronic industries for experimentation.

Nevertheless and thanks the examples of many applications carried out outside Italian's country, we evaluated WiMAX's qualification and its configuration, given from the characteristics of some monitoring network in urban area.

Wimax Forum defined three different physical layers: WirelessMAN SCa, WirelessMAN - OFDMA 256 and WirelessMAN-OFDMA 2048.

WirelessMAN SCa is single carrier interface. WirelessMAN-OFDMA 256 is an interface with multiple carrier: 256 sub carrier realized by using of orthogonal frequencies, the OFDMA (Orthogonal Frequency Division Multiple Access), and TDMA (Time Division Multiple Access). WirelessMAN-OFDMA 2048 assigns a subset of sub-carriers to stations for multiple accessing of their local stations.

In the case of the air pollution monitoring, number of network nodes, bit-rate, modulation, delay link and other parameters define as appropriate physical layer the more common WirelessMAN-OFDMA 256.

\section{Monitoring architecture based on WiFi}

Local area networks can be built in practice, because the assigned frequency bands are licensed and free.

In order to develop software manager that coordinates addressable monitoring structures, including mobile sensors, we analyse and build prototype monitoring architecture using LAN.

The software manager will be based on GIS (Geographical Information System) or better TIS (Territorial Information System) as our platform Terrapack $^{\circledR}[1]$.

It should share data and results elaborated by different remote stations of detection and other similar central stations. In effect, modern information system (as Terrapack) must take care of interface towards other systems and external 
world in general. Interoperability represents a fundamental feature of more efficient management systems that work cooperating together [2,5].

Moreover TIS must include software tools resolving phenomena prediction or it must manage external tools by means of an efficient interoperability [3].

\subsection{COTS device and IP address}

Managed data are numberless and heterogeneous as: air pollution, electromagnetic pollution in urban area, traffic observations, etc. Very different technologies of sensors are often involved. Normally sensor device must be equipped by: ADC (Analog to Digital Converter) devices, electronic control system and I/O (Input/Output) interfaces.

Controller manages: sequence of measures in accordance with the standard programming, storage of data inside local memory before their transmission to remote station or directly to central station, setup of measurement depending by instructions received from an external command (local or remote).

COTS (Commercial Off-The-Shelf) devices are generally used decreasing cost of the complete system. Often I/O interfaces of COTS devices allow connection by means of wires and parallel or serial standards as: RS232, IEEE488/GPIB/HP-IP, etc. In any case these interfaces cannot include IP address. In particular serial I/O connects two devices or systems by point-to-point mode. Network concept together with the distributed architecture is so lost.

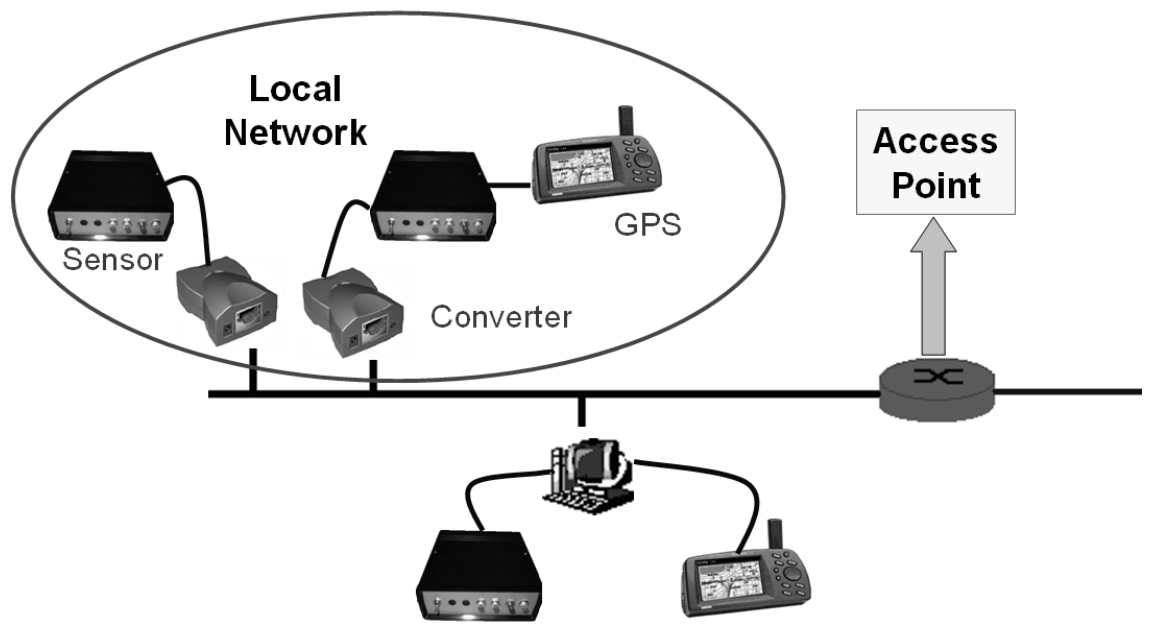

Figure 3: Converters from RS232 to Ethernet I/O allow the inclusion of devices without their HW/SW alteration.

Fortunately, new interfaces have been developed by some electronic industries: they include network board converting from RS232 to Ethernet I/O. No change of hardware and software is necessary. So devices can be controlled remotely without their alteration, replacement of electronic parts and change of 
their original software. Obviously these new components, connected to sensor or device, must be configured by means of special software (and firmware) inside station that directly controls the same device. Tibbo Technology Company produces some model of these converters [4].

Our project includes DS202 Tibbo Devices. A PC sends data to the device (in our case, the sensor) by means of one among: DS202, virtual serial port, UDP or TCP protocol. Virtual serial port can be carried out by using simple Tibbo Technology software. Characteristics of DS202 are: 100/10BaseT Ethernet port, RS232 serial interface, $12 \mathrm{~KB} * 2$ routing buffer size, 10 25 VDC power supply voltage, $60 \times 47 \times 30 \mathrm{~mm}$ mechanical dimensions.

An application firmware installed inside the DS202 allows a considerable number of functions. Firmware consists of two parts: the Monitor and the Device Server Application Firmware. Monitor starts the application firmware and its revision. Device Server Application Firmware provides all functions, in particular the more important: a transparent communication among hosts of network and serial device connected to Tibbo.

\section{Prototype LAN}

In order to test architecture performance, a LAN version has been carried out monitoring remote area. Sensors have been installed for measuring physical parameters as temperature and pressure. Relative position of webcams realizes a wide area of monitoring into a part of a city. Command signals are used for the setup of remote devices: special sequence of measures, frequency of detection, direction and tilt adjustment of the webcam, etc.

We used serial-to-Ethernet I/O converters for working with all server devices identified by IP addresses.

Firewall and gateway problems of transmission are solved by a LinkServer application when a lot of nodes make up the network. Communication is established at first with a server where LinkServer is running and subsequently from the last one server to device server. For a few of nodes a Device Server Toolkit (DST) can be used. So, one user interface as been realized including some tools of DST: Device Server Manager and Virtual Serial Port Manager. This interface lists all devices connected in the network. It shows characteristics of device server as: typology of data source, number of COM port, bit-rate of transmission, file name of data. Two different type of working have been activated: static and dynamic. In the static mode we interrogate device manually. Dynamic mode processes automatically all devices included in an arranged beforehand list. It is the working modality generally activated for controlling many sensors and for receiving information data in a specified input file. Format and parameters of received data are pre-defined from user by means of DST.

Prototype architecture has been tested also into a different environment and activity: the remote control of a rural farm with high level of technology.

Wireless connections of new devices server are including. For this extension, a simple revision of user interface that manages all remote stations (connected by wires or linked by wireless) must be made. 


\section{Conclusions and acknowledgment}

New technologies of ICT are changing the project line of environmental monitoring and remote control of devices spared in a wide territory. In particular wireless technology evolution can allow NLOS links when distance from controller and remote devices is also large. Management of big number of network nodes is achieved. So, metropolitan area could be monitored for developing integrated activity of air pollution activity, motor traffic, etc.

The essential advantages of this approach are the use of IP addresses with Ethernet $\mathrm{I} / \mathrm{O}$ interface and Internet utilization for sharing data and remote controls.

We thank Eng. Francesca Persi for the development of user interface by JAVA and for the test activity of LAN version of the project.

\section{References}

[1] Ferrara V. \& Ottavi, C.M., "Structures and organisation of an information tool dedicated to simulation and management of environmental risks", RISK ANALYSIS III, WIT Press, pp. 213-222, 2002

[2] Ferrara V. \& Guerriero M., "Territorial Information System interoperability: a design improving interaction in an emergency", Risk Analysis IV, Wit Press Southampton, Boston, pp. 475-484, 2004

[3] Ottavi, C.M., Ferrara, V., Guerriero, M., "Prediction method that extends the point to point procedures to large areas in the evaluation of broadcasting services and interference levels in band IV, V and VI", ALTA FREQUENZA, LVIII C(2), pp. 217-223, 1989

[4] "Tibbo Ethernet - to - Serial Devices: Hardware, Firmware, PC Software", www.tibbo.com

[5] V. Ferrara, "Integrated data and utilities to support sustainable planning", Sustainable Planning \& Development, Wit Press Southampton, Boston, pp. 375-382, 2003

[6] V. Ferrara, C.M. Ottavi, "Technology based on distributed GIS tools: an approach for sustainable planning", Sustainable Development and Planning II, Wit Press Southampton, Boston, Vol. 2, pp. 785-794, 2005

[7] RTCM Special Committee No. 104, “ RTCM standard 10401.1 for Differential Navstar GPS Reference Stations and integrity monitors (RSIM)", Arlington, Virginia, USA, August, 2001

[8] Parkinson, B.W., Spilker Jr., J.J., "Global Positioning System: Theory and Applications - Vol. II", Progress in Astronautics and Aeronautics - Paul Zarchan Ed., Vol. 164, 1996

[9] Gebhard, H., Weber, G., "Networked Transport of RTCM via Internet Protocol", Design-Protocol-Software, published by Federal Agency for Cartography and Geodesy, June, 2003

[10] IEEE Standards 802.1la, 802.1lb, 802.1lg

[11] IEEE Standard 802.16 
408 Sustainable Development and Planning III

[12] Liangshan, M., Dongyan, J. "The Competition and cooperation of WiMAX, WLAN and 3G", International Conference on Mobile Technology, Application and Systems, Guangzhou, China pp. 1-5, 2005

[13] Ferrara, V., Gariazzo, C., Ottavi, C.M., Poli, U.,“ An Integrated approach to the pollution hazard analysis and management", RISK ANALYSIS II, WIT Press, pp. 23-32, 2000 